África (São Paulo, 1978, Online), São Paulo, n. 42, p. 61-76, 2021

http://dx.doi.org/

\title{
L'afrique dont on ne parle pas
}

\section{OLABIYI Yai'}

Résumé: Le theme de ce colloque est très important et original. Mais avant toute chose et par fidélité à l'Afrique il me semble qu'outre thème devrait nous inciter à commencer par nous poser des questions sur la philosophie et l'éthique de la parole en Afrique Et c'est cette éthique africaine millénaire qu'il s'agit, aujourd'hui, de réhabiliter et de rétablir dans tout discours sur l'Afrique.

Mots-Clés: La parole africaine ; Les langues africaines

Resumo: $\mathrm{O}$ tema desta conferência é muito importante e original. Mas antes de mais nada, e por lealdade à África, parece-me que, além deste tema, devemos começar por nos questionar sobre a filosofia e a ética do discurso na África. E é esta ética milenar africana que hoje, é uma questão de reabilitar e restaurar sobre qualquer discurso sobre a África.

Palavras-chaves: A palavra africana; As línguas africanas

Nos assises feront date dans les annales de ce que, de par le monde, les universités nomment "Études Africaines". On parlera de ce colloque comme de "l'Initiative de Sao Paulo" ou du "Tournant de l'USP". Ce n'est là ni vaine vaticination, ni déclaration de suffisante autosatisfaction. IL S'AGIT PLUS SIMPLEMENT D'UNE OPINON ÉTAYÉE PAR LE FAIT

\footnotetext{
Texto de abertura da Conferência África de que não se fala, promovida pelo CEA-USP.

2 Ex-Embaixador da República do Benin junto a ONU/ Presidente do Conselho Executivo da UNESCO
} 
QUE C'EST LA PREMIÈRE FOIS À NOTRE CONNAISSANCE QUE, DANS L'HISTOIRE DES ÉTUDES AFRICAINES, UNE INSTITUTION PROPOSE UN THËME AUSSI ORIGINAL, INATTENDU, INSOLITE AU SENS LE PLUS LAUDATIF DU TERME, ÉNIGMATIQUE, PROVOCATEUR MÊME ET GROS DE DÉBATS DONT ON NE PEUT SAVOIR/PRÉVOIR LABOUTISSEMENT. : "l'AFRIQUE DONT ON NE PARLE PAS."

En règle générale, les Études Africaines nous ont habitués à des thématiques plutôt convenues, classiques, parfois même dictées par l'air du temps ou les circonstances ou convenances politiques, lorsqu'elles ne sont pas suggérées par les pouvoirs politiques, les fondations et autres instances ou lieux de financement de projets. Le CEA de l'USP mérite donc d'autant plus nos félicitations pour cette heureuse initiative ;

L'originalité de notre intitulé : 'L'AFRIQUE DONT ON NE PARLE PAS/A ÁFRICA DE QUE NÃO SE FALA est telle que, d'entrée de jeu et à l'inverse du protocole universitaire qui préfère réserver les suggestions à la fin des communications, je m'empresse de faire une recommandation que j'estime d'importance : à savoir que nos assises, à travers le CEA-USP, suggèrent à toutes les associations d'études africaines( en Grande Bretagne, en France, en Allemagne, la JASA au Japon, en Chine, l'Association Canadienne des Études Africaines la puissante African Studies Association-ASA aux USA et bien évidemment, sur le continent lui-même, l'Association Africaine des Études Africaines,) qu'elles inscrivent à l'agenda de chacune de leurs réunions, quelle qu'en soit la périodicité , une table ronde ou un atelier sur "l'AFRIQUE DONT ON NE PARLE PAS".

Mais avant toute chose et par fidélité à l'Afrique il me semble qu'outre thème devrait nous inciter à commencer par nous poser des questions sur la philosophie et l'éthique de la parole en Afrique. Je prendrai mon exemple de ma culture, la culture yoruba, par bonheur très connue et même très influente sous nos cieux brésiliens où elle est désignée surtout comme Cultura nagô. Mais il est bon de garder à l'esprit que cette philosophie est partagée largement, dans son essence, malgré des variantes, par toutes les cultures africaines. Voici ce que dit de la parole Ifa, le corpus de la sagesse yoruba, dans son "chapitre" nommé Òsá-Gudá :(Abiodun :26-31) 
La parole est sacrée. Elle n'est pas au début, comme dans la tradition judéo-chrétienne. La parole est fille de Olodùmarè, la divinité créatrice. Et du sein de Olodumare, elle demande à naître et grommelle (Oro hùnnùhùnnù). La divinité créatrice la mit au monde en la vomissant(Hòòrò) de là-haut, en quelque sorte sous forme de pluie. La Parole dans le ventre de la divinité, est ainsi présentée comme impatiente et impure (ce qui nous fait penser aux 'Impurezas do branco de l'inégalable Carlos Drummond de Andrade). C'est en sortant du sein de la divinité créatrice qu'elle se purifie. Pour la purifier, Olodumarè l'a alors mâtinée de trois éléments :

\author{
Ogbon, (la sagesse) \\ Imo (le savoir) et \\ Oye (la faculté de compréhension/discernement)
}

C'est alors qu'ORO, LA PAROLE, reçut la permission de descendre sur terre. (Abiodun, 2014 : 26-36). Il faut en conclure qu'en Afrique, dans nos traditions intellectuelles, philosophiques et discursives, la parole est précieuse. C'est une affaire sérieuse, sacrée même, puisqu'elle est fille et don de Olodumarè, le Dieu créateur. On n'en use pas à tort et à travers. Il importe également de retenir que la sagesse, le savoir et le discernement en sont les principales composantes, les principaux ingrédients sans lesquels son avènement au monde serait comme fantomatique, irréel un faux en quelque sorte. D'autres cultures africaines assimilent la parole à un œuf, élément si précieux et fragile qu'il faut se garder de le laisser tomber, sous peine d'en perdre à jamais l'essence, l'élément qui lui permet de se renouveler.

Parce que la parole est sacrée, en faire usage est un acte de responsabilité et de purification envers soi et envers celui ou celle à qui cette parole est adressée. C'est un acte quasiment religieux dont il importe de ne pas abuser. C'est cette attitude de respect quasiment religieux de la parole qui a déserté les lieux où et les personnes qui s'arrogent le droit de parler de l'Afrique et pour l'Afrique, ceux-là qu'Aimé Césaire désignait de « ravisseurs du Mot », et « détrousseurs de la Parole ». Et c'est cette éthique africaine millénaire qu'il s'agit, aujourd'hui, de réhabiliter et de rétablir dans tout discours sur l'Afrique, qu'il vienne des journalistes ou des africanistes des plus grandes universités du monde. 
Le premier obstacle sur le chemin de cette nécessaire réhabilitation est l'exclusion des langues africaines des lieux de production des discours de tous genres sur l'Afrique, ce qui fait que la voix de l'Afrique profonde n'est que rarement entendue .Je soulève là un immense problème, qui est à la base de la thématique qui nous occupe, à savoir "l'Afrique dont on ne parle pas". Nous touchons là à un aspect d'un concept que j'aimerais appeler l'EXCEPTION AFRICAINE. Celle-ci a plusieurs dimensions. Celle qui nous intéresse ici est que c'est toujours quelqu'un d'autre, un certain "ON" qui parle de l'Afrique. Notre continent ne parle presque jamais. C'est le seul continent dont aucune langue n'est parlée dans les hautes sphères de décisions du monde (ONU, UNESCO, FAO...etc). L'exception africaine, c'est qu'il existe des journalistes spécialistes de le l'Afrique et de grande réputation qui ne peuvent pas faire une phrase dans une langue africaine, qui ne lisent pas une seule ligne d'une langue africaine. Imagine-t-on un journaliste de $\mathrm{La}$ PRAVDA, de LE MONDE, ou du NEW YORK TIMES, spécialiste du Brésil et qui ne parle pas portugais ? Ou un spécialiste brésilien de la France du temps du général de Gaulle qui ne parle et ne lise pas le français? Comment dès lors entendre la voix de l'Afrique profonde, millénaire, celle, ne l'oublions pas, où a commencé la civilisation humaine, lorsque, à chaque fois, la voix des Africains doit transiter par le filtre ou le prisme déformant d'une langue-pensée, d'une langue-culture non-africaine ? Il y a là comme une manière de "fourches caudines" (forcas caudinas) imposées à la parole africaine. IL n'est pas étonnant que, l'Afrique profonde étant privée de la parole et de son éthique de la parole telle qu'esquissée plus haut, ceux qui ont usurpé sa parole laissent dans le silence l'essentiel de son être, de ses valeurs et aspirations. Pour tout dire, IMENSA É A ÁFRICA DE QUE NÃO SE FALA. Je voudrais nous inviter, en tant que linguiste, et Africain, e tant qu'être humain, tout simplement, à prendre très au sérieux ce problème de l'exclusion des langues africaines des sphères de l'officialité et de la blogosphère. Car si la faculté de langage est ce qui distingue les animaux de l'homme, la langue est le premier droit humain. Par conséquent, nier sa langue à une personne est lui nier son statut de personne humaine. C'est le ravaler au niveau d'un animal. C'est bien là, vous vous en souviendrez, l'attitude de Prospero envers Caliban, dans la Tempête, de Shakespeare 
L'archéologie de cette usurpation de la parole pourrait nous faire remonter à Pline l'Ancien, avec un tropisme animalier. En effet, dans son Histoire Naturelle, ce rhéteur latin disait en substance que 'à propos des scorpions, personne en Afrique ne "entreprend quoi que ce soit sans avoir auparavant prononcé le mot Afrique" (Livre V, 2). Ce tropisme animalier Plinien perdure jusqu'à nos jours, et l'attitude des faiseurs d'images de l'Afrique, ces détrousseurs de la parole africaine est, à y bien réfléchir, une variation sur le même thème. ((IMAGES COMPARÉES DE LAFRIQUE ET DES AUTRES CITÉS ET DESTINATIONS POUR TOURISTES DANS LE MONDE : PARIS, BRUXELLES, ROME, ATHÈNES, NEWYORK...RIO DE JANEIRO, L'ICÔNE MANDELA OUBLIÉ QUI AURAIT PU IMMORTALISER LE SYMBOLE D'UNE VALEUR UNIVERSELLE)).

Je voudrais à présent commenter la richesse sémantique de notre intitulé: A ÁFRICA DE QUE NÃO SE FALA est une phrase aux multiples interprétations et qui suscite de nombreuses interrogations :

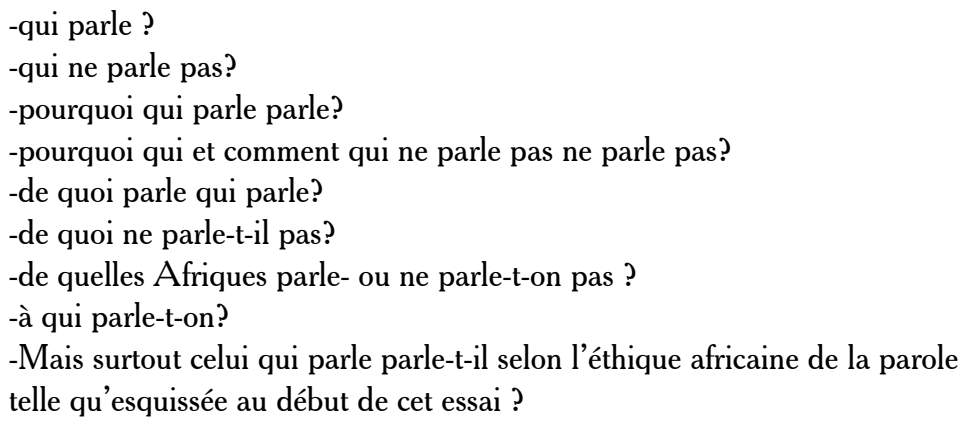

On se rend donc compte que notre thématique est, pour ainsi dire, tout un continent.

Au degré zéro de l'interprétation, la phrase A ÁFRICA DE QUE NÃO SE FALA pourrait s'interpréter comme visant des choses qui se réalisent ou des évènements qui se produisent en Afrique et dont on ne parle jamais ou si peu. Mais, même ce degré zéro est passible d'interprétation binaire:

A) des choses positives dont on ne parle pas

B) des choses négatives qu'on cache. 
Citons rapidement des exemples de

\section{A) Des choses positives dont on ne parle pas :}

1. Chaque jour il se créé en Afrique des dizaines de coopératives, surtout de femmes pour lutter contre l'exclusion et la pauvreté. Elles se constituent en groupes de "'èsúsú' ou tontines, bien avant l'invention du micro-crédit par le prix Nobel bangladais Yunus. Les journalistes économistes de l'Occident taisent cette dimension fondamentale de l'économie et de la vie africaine que les spécialistes paresseusement rangent sous l'inopérant concept d'économie informelle.

2. Je me dois également de citer le cas d'autres, femmes à un grade supérieur de l'économie de marché, les fameuses 'MUMMY BENZ' qui font le commerce de longue distance, de n'importe quel aéroport de l'Afrique occidentale ou centrale vers Dubai, Delhi, Beijing, Bangkok ou Shanghai. CES AFRICAINES GÉNIALES ONT L'ART DE PERCEVOIR CE QUI SE VEND OU QUI PEUT SE VENDRE LE MIEUX ENTRE L'AFRIQUE ET LE RESTE DU MONDE, EN DEHORS DÉRIVÉS DU COMMERCE D'EXTRACTION DE NOS RESSOURCES NATURELLES QUI EST L'APANAGE DE L'ÉCONOMIE CLASSIQUE AFRICAINE DONT LES ÉCONOMISTES OFFICIELS FONT L'ESSENTIEL DE LEURS DISCOURS ÉCONOMIQUES SUR L'AFRIQUE.

3. L'Afrique dont on ne parle pas, ce sont les petites couturières et les brodeuses à peine scolarisées, mais qui créent chaque jour des trésors en fait de mode africaine.

4. Des coopératives se forment le long du fleuve Niger, à Bamako, Gao, Tombouctou, Niamey, pour planter des arbres, résister à l'avancement du Sahara et à l'ensablement conséquent du fleuve Niger. Les journalistes et autres spécialistes africanistes ne s'intéressent pas à ces initiatives pourtant vitales pour les populations africaines.

5. Plus important, sous cette rubrique d'évènements positifs dont on ne parle pas est la créativité des jeunes africains. Il existe des milliers de jeunes inventeurs africains, qui d'ailleurs, sur le continent, s'organisent et se mettent en réseaux. Les initiatives sont les plus nombreuses dans les domaines de l'électronique et de l'informatique. Pas de semaine sans un nouveau logiciel 
pour résoudre des problèmes africains. Il y'a à peine un mois, le jeune congolais Arnaullt MUKENGE inventait un jeu vidéo dans un but social: démolir Zuma. Il entendait ainsi apprendre aux jeunes africains à envisager, plus tôt que tard, de se débarrasser des hommes politiques africains corrompus qui empêchent nos peuples d'avancer. Cette invention vaut autant que notre colloque.

6. S'agissant toujours de l'Afrique dont on ne parle pas et qui mérite qu'on en parle universellement, comment ne pas mentionner la foisonnante littérature africaine en langues africaines malgré le peu d'intérêt qu'elle suscite de la part des officiels ? De véritables chefs d'oeuvre s'écrivent dans tous les genres en hausa, yoruba, kiswahili, cicewa, zulu, ewe, wolof, amharique, gikuyu et qui disent toutes les facettes de la vie africaine d'hier d'aujourd'hui et de demain. Ces oeuvres n'obtiendront aucune reconnaissance internationale ni Prix Nobel, ni Prix Goncourt, Renaudot, Cervantes ou autres, parce que les juges ne lisent pas nos langues. Mais elles n'en sont pas moins africaines, belles et de valeur universelle. Il faut espérer qu'elles seront un jour "découvertes" par l'Occident, et le reste du monde, lorsque nos peuples se seront battus pour imposer des relations culturelles et linguistiques plus équitables dans notre mondialisation encore bancale. Imaginez ce que l'humanité aurait perdu, si l'Indien Rabindranath Tagore n'avait pas été « découvert » et traduit !

7. L'Afrique dont on ne parle pas et dont la voix est si belle et sonore, est celle de la créativité de nos poètes oraux, dans les langues africaines comme dans celles européennes ou métissées des villes, et dont la palette toujours grandissante et mouvante, va des genres oraux dits "traditionnels" au hip hop, au slam, au rap.

8. A África de que não se fala é sobre tudo a esquisita poesia oral das mulheres africanas. Ela é uma sabia e estética dosagem de mel e fel de maneira a esquivar a censura de um público literário geralmente machista e androcentrista.

Permettez-moi à présent d'aborder également des exemples de B), l'autre bout de l'interprétation binaire du degré zéro de notre intitulé, à savoir Des choses négatives dont on ne parle pas, dont l'Occident choisit de ne pas parler, par intérêt. 
Il s'agit en premier lieu, des turpitudes de la plupart de ceux qui nous dirigent en Afrique, des faits et gestes les plus négatifs la classe kleptocratique africaine .Je cite pêle-mêle:

1. le bradage de nos ressources du sol, du sous-sol, de la mer, et de la terre, et la trahison des intérêts africains.

2. Beaucoup de gouvernements africains (L'Ethiopie vient en tête), avec la complicité de la Banque Mondiale vendent les terres africaines à des magnats arabes (du Golfe) et chinois qui y font de l'agriculture intensive et réduisent à l'état de simples prolétaires agricoles ceux qui depuis des millénaires vivent sur ces terres ; Il s'agit là d'une forme lente de génocide. Il s'agit également d'épistémicide (Sousa Santos, 2016) puisque les savoirs agricoles ancestraux sont ainsi réprimés et exclus du champ des pratiques agricoles. Si, à terme, il n'est pas mis fin à ce phénomène de bradage de nos terres, les Africains deviendront des étrangers sur leur propre continent. $\mathrm{Ce}$ phénomène important de bradage des terres a fait l'objet du documentaire "DEAD MONKEYS FEAR NO HYENAS" par le cinéaste suédois Joakim Demmer.

3.Il y'a à peine deux semaines, la presse nigériane "révélait" au public la découverte, dans un compte bancaire domicilié aux USA, 12 millions, 831173 Dollars USA dans le compte d'une ex-première dame, ainsi que 7, Millions 900 mille dollars dans le compte de sa mère qui venait de mourir. Fait divers au Nigeria, mais commun dans les pays africains. Les journalistes et africanistes de réputation mondiale ne le tiennent pas pour significatif.

4. Sur le plan continental à présent : les pères des indépendances africaines ont eu la sagesse de créer l'OUA (ORGANISATION DE L'UNITÉ AFRICAINE) en 1963. Cette organisation est, depuis devenue l'UNION AFRICAINE(UA). Ce dont on ne parle pas de cette organisation, bien qu'il s'agisse d'un secret de Polichinelle, c'est que:

a. Son siège, à Addis Ababa, a été construit et donné en cadeau à l'Afrique par la Chine. Une organisation qui dirige tout un continent aussi riche que l'Afrique et incapable de se construire un siège, après un demi-siècle d'existence! Peut-on prendre au sérieux cette institution et ceux qui en sont membres? 
África (São Paulo, 1978, Online), São Paulo, n. 42, p. 61-76, 2021

b. Pire, les deux tiers des salaires des fonctionnaires de l'Union Africaine sont payés par.........l'Union Européenne. !!????. N'est-ce pas là une belle performance de nos pseudo-bourgeoisies africaines après plus d'un demi-siècle d'indépendance? Comment donc ne pas en parler?

Nous pourrions ainsi allonger, ad infinitum, la liste des turpitudes de la classe dirigeante kleptocratique africaine, mais je préfère éviter de faire la chronique de ces bousiers, pour, à présent dire quelques mots, puisque cela m'a été demandé, de l'état de la démocratie en Afrique, dans le contexte de l'Afrique dont on ne parle pas.

À l'indépendance, les états africains, même ceux qui étaient des 'royaumes', ont tous adopté la démocratie de type euro-américain comme modèle d'état. Il n'y avait d'autre justification à cette adoption que le fait que la démocratie est un 'héritage'(??)colonial, les colonisateurs eux-mêmes l'ayant hérité de la Grèce. LA DÉMOCRATIE NOUS A DONC ÉTÉ DONNÉE COMME UN MODĖLE UNIVERSEL DE GOUVERNEMENT, DANS LE BAGAGE EMPOISONNÉ DES PSEUDO-INDÉPENDANCES. Mais qu'en est-il, en réalité, de ce modèle présenté comme un instrument propre à nous assurer le 'rattrapage' de l'Europe, de cet instrument prétendument universel dans les mains des nouvelles élites gouvernantes africaines? La démocratie, en Afrique au début des indépendances, s'est résumée en l'imitation sans critique des modèles que chaque métropole européenne offrait à sa colonie : modèle parlementaire Westminsterien, mono ou bicamérale, système présidentiel, séparation des pouvoirs, élections au suffrage universel. Ces modèles étaient censés aider les Africains à construire des états-nations sur le modèle européen. L'exercice, la tâche de " nationale », ce véritable éléphant blanc mené sous le contrôle et avec la bénédiction des maîtres européens, a conduit presque invariablement aux régimes de partis uniques, dont les représentants les plus ubuesques sont l' " empereur » Bokassa, le maréchal Idi Amin Dada le maréchal Mobutu Sese Seko ..etc., les régimes les moins ubuesques n'en étant pas moins intolérants, répressifs et parfois sanglants, tout en se parant du nom de démocratie, au vu et au su de leurs maîtres européens. Les gouvernements qui se sont réclamés d'obédience 'démocratie socialiste' ne sont guère différents en essence: partis uniques, répression, embrigadement de la jeunesse, des femmes et des 'masses populaires' en général. Seul le contexte de soulèvement et de protestations 
populaires enclenché par la chute du mur de Berlin et la fin des démocraties de type soviétique en Europe de l'Est a forcé les puissances coloniales européennes à amener leurs protégés africains à réviser leur expérience démocratique et de construction nationale. Ce fut alors l'ère des 'Conférences Nationales Souveraines' inaugurées au début des années 1990 du siècle dernier avec plus ou moins de bonheur selon les pays. Nous disposons à présent de près de 30 ans de recul après ces expériences et pouvons donc les juger. Certes, on peut dire que les 'régimes de renouveau démocratique' comme on les nomme, issus de ces conférences, constituent une avancée par rapport à ceux de partis uniques nés des premières expériences démocratiques. Mais ces succès sont mitigés et la différence est plus de degré que de nature. L'émergence de la société civile africaine ces trois dernières décennies est certainement le gain démocratique le plus appréciable : mouvements de femmes, de jeunes (du type Le Balai Citoyen, $Y$ en a marre.etc) nés un peu partout et qui défient les gouvernements autocratiques et les dictateurs. Certes, il y'a de véritables mais rares histoires de succès comme le Botswana, Maurice, le Ghana, le Bénin, le Cap Vert et le Rwanda (et à quel prix pour ce dernier !). Mais pouvait-il en être autrement dans le contexte de la chute de l'apartheid, de l'avènement du mouvement altermondialiste global, de l'éveil des peuples latino-américains, du Printemps arabe et autres mouvements citoyens de par le monde? Malgré des avancées indéniables, la seconde vague de la démocratie en Afrique s'est révélée finalement non soutenable, et en réalité un échec, si l'on se met du point de vue de ce que les peuples attendent. Parmi ses nombreuses insuffisances, il faut citer :

-sa non-inclusivité. La société civile qui est l'un de ses gains les plus importants est essentiellement un phénomène urbain. Les campagnes continuent d'être marginalisées comme par le passé.

-Il lui manque, tragiquement, une dimension ou un contenu économique, social et culturel. , l'attention étant concentrée sur le 'fonctionnement normal des institutions'.

-Précisément parce qu'elle est surtout une démocratie formelle, non-inclusive et sans contenu socio-économique réel, les classes dirigeantes africaines n'ont eu aucun mal à manipuler à leur guise les élections pour se perpétuer au pouvoir. Celles-ci (les élections) ne sont en général que de grandes farces ou de grandes kermesses d'achat de voix et de corruptions auxquelles, pour 
donner le change, sont conviés les peuples africains à intervalles plus ou moins réguliers.

-Son talon d'Achille, son péché originel, congénital, qu'elle partage avec celle de la première période des indépendances est qu'elle nous est venue d'ailleurs, imposée ou à tout le moins suggérée par nos maîtres (Discours de La Baule du Président français François Mitterrand). En clair, CE QU'ONNE DIT PAS DE LA DEMOCRATIE EN AFRIQUE EST QU'ELLE EST PAR ESSENCE, ET $a b$ ovo, « LA DÉMOCRATIE DES AUTRES » pour emprunter une expression à l'économiste Amartya Sen. Nous ne nous sommes pas approprié la démocratie en faisant œuvre de souveraineté et de responsabilité intellectuelle, en pensant, comme dit un jeune africain, du passé des autres notre avenir. Cette démocratie des autres a donc été une greffe qui n'a pas réussi. Me vient à l'esprit une foule de proverbes pour caractériser notre situation :

Quien lo suyo deja neciamente

Busca lo ajeno malamente ( espagnol)

\author{
Àgbàbò sòkòtò \\ Bí kòfún ni nígun \\ A so ni nídíi (yoruba) \\ (le pantalon d'emprunt \\ sois-vous serre au tibia \\ ou est trop ample à la ceinture).
}

C'est donc 'là que le bât blesse'. 'Si le colis du pied bot est penché, dit un proverbe yoruba, la faute n'est pas à sa tête, regardez plus bas', (Amókùnún, erù re wó - Ó ní 'òkè le ń wò')

Il nous faut donc prendre une pause et réfléchir. Je nous recommande, pour ce faire, les paroles suivantes d'un des meilleurs fils de l'Afrique de la diaspora, de l'Afrique globale, j’ai nommé Aimé Césaire :

(i)

La voie la plus courte et la plus sûre vers l'avenir passe toujours par l'approfondissement du passé"

(ii) 


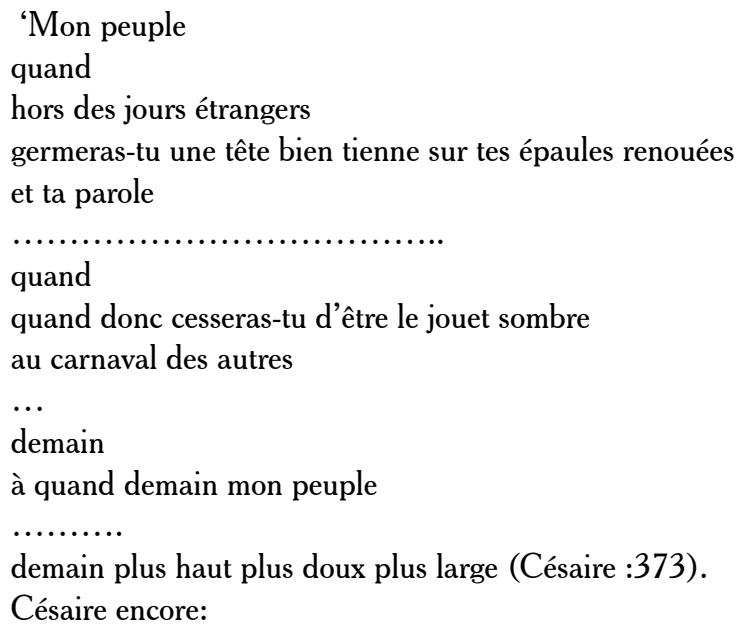

(iii)

La naïveté est d'attendre qu'une voix, je dis bien qu'une voie vous dise: par ici la sortie!

(iv)

La relance ici se fait par le vent qui d'Afrique vient la relance ici se fait par l'influx plus encore que par l'afflux.

Marquons donc une pause et réfléchissons un moment:

Remontons aux sources de la démocratie, qui, comme l'on sait, est grecque. Ce système de gouvernement, qui nous est présenté comme modèle universel, excluait dans son berceau en Grèce, les femmes et les esclaves. N'est-ce pas faire preuve d'une suprême naïveté, nous Africains qui avons tant souffert de l'esclavage, que d'attendre d'un tel système un potentiel magique d'émancipation ? N'est-ce pas au nom de cette même démocratie, que Napoléon Bonaparte a emprisonné Toussaint Louverture au fort de Joux jusqu'à ce que mort de froid s'ensuive. N'est-ce pas ce même Napoléon qui voulait et voulait rétablir l'esclavage à Haïti? La démocratie européenne ne 
África (São Paulo, 1978, Online), São Paulo, n. 42, p. 61-76, 2021

s'est-elle pas toujours accommodée des conquêtes coloniales, du colonialisme, du racisme, du néo-colonialisme et de l'apartheid ? Ne voyons-nous pas aujourd'hui, sous nos yeux, dans son lieu de naissance, la Grèce de Tsípras, cette démocratie écraser le peuple grec en lui imposant de lourds sacrifices à travers un gouvernement que ce même peuple, DÉMOCRATIQUEMENT, élu ?

De ces interrogations, il faut bien conclure que la démocratie telle qu'elle nous a été léguée en Afrique, a échoué et ne pouvait qu'échouer. Que, s'agissant de l'Afrique et de la démocratie, ce dont on ne parle pas c'est de L'URGENCE D'INVENTER NOUS-MÊMES UN SYSTÈME DÉMOCRATIQUE ENDOGÈNE À PARTIR DE NOTRE HISTOIRE, DE NOS CULTURES ET DE NOS VALEURS. De ce point de vue, l'Afrique est à la croisée des chemins. «L'heure de nous-mêmes a sonné ». C'est dans cette nouvelle direction que s'oriente la jeunesse africaine de nos jours. D'où la nécessité d'une plongée dans notre histoire plusieurs fois millénaire pour y recenser les philosophies politiques et les textes anciens, les « épistémogonies » (Zé Belinga) régissant le vivre ensemble africain dans notre passé précolonial et anti-esclavagiste, aux fins de les étudier de façon critique, SANS CULTURALISME, et d'en tirer des pépites.

La philosophie UBUNTU : Je suis parce que nous sommes' (Ramose), LA CHARTE DE KURUKAN FUGA, contemporaine de la MAGNA CARTA de l'Angleterre, le KER ISSA sont des exemples d'épistémogonies dont il est bon de s'inspirer. Les cultures africaines sont riches de tels textes. Notre défi est de les sortir de l'oubli, de les étudier de façon critique, de les approfondir et de nous en inspirer pour solutionner les problèmes de l'Afrique contemporaine. Permettez-moi de citer, à titre d'exemple, quatre principes ou articles de LA CHARTE DE KURUKAN FUGA qui me semblent être d'une grande pertinence de nos jours:

1. Konenbeenin : "Toute vie est une vie. Chacun a droit à la vie et à la préservation de son intégrité physique.

2. N'offensez jamais les femmes, nos mères.

3. Les femmes, en plus de leurs occupations quotidiennes, doivent être associées à tous nos gouvernements 
4. Avant de mettre le feu à la brousse, ne regardez pas à terre, levez la tête en direction de la cime des arbres pour voir s'ils ne portent pas de fruits ou de fleurs.

\section{CONCLUSION}

L'Afrique dont on ne parle pas est vaste et profonde. Elle vit, grouille, se parle, créé, produit, chante, rit et danse. Notre défi est de créer des instances de prise de parole pour faire entendre la parole de l'Afrique profonde, défaisant ainsi les stéréotypes et clichés des 'ravisseurs du mot' africain, et des 'détrousseurs de la parole' africaine. Je voudrais pour terminer, réitérer et expliciter ma suggestion faite au début de cette présentation, celle que je voudrais encore une fois, nommer LE TOURNANT DE L'USP dans les ÉTUDES AFRICAINES: qu'à chaque réunion des associations d'études africaines il s'organise une Table Ronde autour du thème 'L'AFRIQUE DONT ON NE PARLE PAS' . Qu'à ces tables rondes on fasse venir, comme participants privilégiés, des Africains et surtout des Africaines de l'Afrique profonde. Comme il se peut fort bien qu'ils ou elles ne parlent aucune des langues européennes de nos réunions, qu'on leur assure une traduction par des Africains bilingues-biculturels solides et avérés. Il s'agit là d'un geste épistémologique novateur dans les études africaines que j’encourage le CEA-USP à accomplir. Ainsi commencera à s'entendre la voix de l'Afrique profonde.

Notre continent, l'Afrique, est la sœur aînée du monde, puisqu'elle est le berceau de l'humanité. Mais elle est également la sœur cadette du monde, parce qu'une grande partie des enjeux de l'avenir de l'humanité se jouera sur le continent africain. C'est pourquoi, en toute sagesse, l'Afrique a la patience de supporter qu'on nenparle pas d'elle. Mais pas pour longtemps, car sa jeunesse ne tardera pas à révéler le vrai visage, si mal connu de l'Afrique.

Pline le Jeune, le neveu du naturaliste qui ne voyait que des scorpions en Afrique, disait, au contraire de son oncle :

'Ex Africa semper aliquid novi' (De l'Afrique, il faut toujours s'attendre à une surprise). 
Et il a raison, comme en témoignent deux grands poètes qui comprennent l'Afrique et dont, en manière de viatique, je vous cite des paroles profondes, que je vous invite à méditer.

Il s'agit de Rabindranath Tagore, dans son poème intitulé Africa :

In a insane time, the long, long past, When the Creator himself gravely displesased himself, Destroyed his own creation over and over again, The ocean with its angry arms, snatched, Away a piece of the eastern earth, And calle dit Africa..........

Alas, Africa of shadows, your human face remains unknown to the darkened vision of contempt.

They came

Human hunters all.

The iron chains, and claws sharper than wolves.

Their pride blinder than your sunless forests.

The barbaric lust of civilized men Revealed in ugliness of their own Inhumanity.

Et d'Aimé Césaire, dans un poème intitulé : pour saluer le TiersMonde :

Je vois l'Afrique multiple et une

Verticale dans la tumultueuse péripétie

Avec ses bourrelets, ses nodules,

Un peu à part, mais à portée

$\mathrm{Du}$ siècle, comme un cour de réserve.

Vois :

L'Afrique n'est plus au diamant du malheur

Un noir cour qui se trie ;

Notre Afrique est une main hors du ceste, c'est une main droite, la paume devant 
OLABIYI YAI L'afrique dont on ne parle pas

\author{
et les doigts bien serrés ; \\ c'est une main tuméfiée \\ une-blessée-main-ouverte, \\ tendue, \\ brunes, jaunes, blanches, \\ à toutes les mains, à toutes les mains blessées \\ du monde.
}

